\title{
Color Marker Search System using a Pan-Tilt-Zoom Camera for Intelligent Worker Assistance
}

\author{
Yoshinari MORIO $^{1 *}$, Makoto MIWA ${ }^{1}$, Katsusuke MURAKAMI ${ }^{1}$
}

\begin{abstract}
In this study, we developed an autonomous color marker search system that uses a single pan-tilt-zoom camera to detect the $3 \mathrm{D}$ locations of multiple markers placed in a field. The system was designed to scan for multiple markers over a sensing range within $40 \mathrm{~m}$ in a field divided into 855 marker search grids. In the experiments, our system was able to robustly measure marker $3 \mathrm{D}$ positions within a sensing range of approximately $30 \mathrm{~m}$, and with a root mean square error of less than $0.5 \mathrm{~m}$, without large height differences between markers and camera, and without marker deformation, with the maximum marker search time required to scan the entire field of approximately $7 \mathrm{~min}$.
\end{abstract}

[Keywords] color marker search, pan-tilt-zoom camera, intelligent worker assistance

\section{Introduction}

The Japanese agricultural sector faces a number of serious problems including depopulation, a rapidly aging workforce, the extreme physical workload required, and hazardous working conditions. Consequently, the number of agricultural workers in 2011 had declined to around 1.8 million, in comparison with a total labor force of 65.9 million, a decrease of about 540,000 from 10 years ago. Furthermore, the percentage of agricultural workers over 65 years old is approximately $60 \%$. Japan's unemployment rate in 2011 was approximately $4.6 \%$, and the percentage of non-regular employees has been increasing since the $1980 \mathrm{~s}$. The increasing unemployment and non-regular employment rates among young people are becoming one of the nation's more serious problems. Accordingly, the problems facing the agricultural sector must be addressed while taking into account the workforce imbalance between large cities and rural areas, the generation gap, and the current large number of unskilled workers.

The area of automation technology, which includes autonomous tractors and combine harvester navigation systems equipped with global navigation satellite system devices, along with product harvesting and quality grading systems using machine vision, has seen rapid developments over the past decade. However, while such products and systems reduce the human workload and save time, their utility and applicability is restricted to the fields for which they were designed, and they have no interactive functions that enable collaboration with human workers in other areas. Moreover, such systems provide no opportunities for unskilled workers to work in the agricultural sector or to receive education and training related to agricultural work.

In this study, we propose an intelligent agricultural worker assistance system designed to enable unskilled and semiskilled operators at remote sites to control agricultural vehicles and robots via the Internet. The worker assistance system autonomously controls cameras that track workers and identifies workflows based on worker behaviors. The system also formulates assistance request menus such as the timings, locations, and tasks that need to be performed in order to assist workers, and autonomously sends the requests to operators at remote sites without requiring workers to operate the system. Operators at remote sites then control vehicles and robots to provide assistance to field workers based on their requests. A worker tracking system, a worker behavioral recognition system, worker assistance menus, and a decision-making system are essential in developing a worker assistance system.

In a previous study, we developed a real-time singleagricultural-worker tracking system using image processing (Morio et al., 2012). The tracking system used a single pan-tilt-zoom camera (PTZ camera), uniforms for workers with blue and red color markers measuring $100 \mathrm{~mm}$ by $200 \mathrm{~mm}$, and two types of particle filters to track the markers on the uniforms. In operation, the tracking system was capable of detecting the position of a uniformed worker, as well as her or his standing or sitting posture, within a sensing range of approximately $38 \mathrm{~m}$ (resolution: approximately $1 \mathrm{~m}$ ). This system was also capable of detecting eight separate posture types based on the detected positions of the markers to portray the workers' stances.

As an added function of the worker tracking system, we developed an agricultural working motion detection system

\footnotetext{
${ }^{1}$ Graduate School of Bioresources, Mie University, Japan

*Corresponding author: morio@bio.mie-u.ac.jp
} 
for recognizing worker behaviors using the recognition of eight postures as indicated by the markers on the workers' uniforms (Morio et al., 2014). The motion detection system was able to robustly and separately respond to the targeted motions when the positions and postures of the markers on the workers' uniforms changed depending on worker behaviors. However, when the positions and postures of the markers did not change, the system was not able to acquire any information about worker behaviors.

In order to robustly acquire information about working behaviors, we developed an agricultural worker posture recognition system (Morio et al., 2017). This system used a worker's silhouette and eight postures of the markers on a worker's uniform to recognize a worker's posture. The posture recognition system was capable of identifying 18 specific postures performed by a single worker, for example, walking, turning, bending, squatting, stooping, and moving her/his arms along a furrow in an agricultural field. By using worker posture sequences acquired by the worker posture recognition system, we developed a worker behavioral recognition system to recognize three specific types of worker behaviors, watering, seeding, and harvesting, performed by a single targeted worker along a furrow in a field cultivated for peas. The three behavior types were modeled by 14 hidden Markov models: 10 for harvesting behaviors, 2 for seeding behaviors, and 2 for watering behaviors.

In these previous studies, the worker tracking system, which used a single PTZ camera, was designed to track only the behaviors of a single worker. To track the worker using the tracking system, the worker had to wear a uniform with blue and red markers, stand in front of the camera, and turn her / his body toward the camera so that the markers on the uniform were detected by the particle filters. When the tracking system lost track of a color marker, the worker had to interrupt her/his work and turn her/his body toward the camera again. If the tracking system failed to detect a marker, the system was not able to autonomously search for the worker. Moreover, the tracking system could not track multiple workers simultaneously.

To improve our previous worker tracking system, we developed an autonomous color marker search system that uses a single PTZ camera. We do not use an omnidirectional camera, but instead used a single PTZ camera in this study because the color markers need to be detected robustly by maintaining a high image resolution without distortion at positions further than $10 \mathrm{~m}$ from the camera.

In related studies, Bagherinia and Manduchi developed a fast color marker detection algorithm using a cell phone camera for environmental labeling, for example, as a way-finding aid for the visually impaired (Bagherinia and Manduchi, 2013).
The system of Bagherinia and Manduchi was based on pie-shaped markers with four colored sectors having diameters of $15 \mathrm{~cm}$ that could be placed in key locations in the environment. A visually impaired person was able to search for the markers by orienting the camera phone in different directions. Bagherinia and Manduchi designed color rendering models to robustly detect the marker area under a wide variety of viewing conditions including different viewing distances and angles, illumination conditions, motion blur, and occlusions. However, the system had no function to measure the distance to the marker, and the detectable range of the system was less than $5 \mathrm{~m}$. To detect multiple human beings without using markers, Dalal and Triggs (Dalal and Triggs, 2005) and Dalal et al. (Dalal et al., 2006) used a histogram of oriented gradients (HOG) descriptor to detect pedestrians with partial occlusions and a wide range of variations in posture, appearance, clothing, illumination, and background. In their studies, the HOG descriptor effectively detected human regions and was able to extract major human contours robustly due to the differences in physique and clothing texture. Viola and Jones proposed the AdaBoost algorithm with Haar-like features to detect a human facial region robustly and in real time (Viola and Jones, 2001). Although the HOG feature-based system and the Haar-like feature-based system were able to detect a human region without using special markers, the systems had no function to distinguish the targeted person from other persons, to measure the distance from camera to the target, or to recognize worker postures. Shotton et al. used a single depth sensor of Microsoft Kinect to accurately recognize 3D human poses in real time (Shotton et al., 2011). The human detection range of Kinect is less than $5 \mathrm{~m}$, too small to track workers in a field. Markovic et al. mounted an omnidirectional camera on a mobile robot to track and follow an object (Markovic et al., 2014). Although an omnidirectional camera can observe the surroundings around the camera, the sensing range of the camera without zoom function is too small to track workers.

In this paper, an image processing system to search for markers is introduced and the precision of the system in measuring the positions of markers is evaluated through experiments. The remainder of the paper is organized as follows. Section 2 details the marker search system. Section 3 summarizes the experimental results and discussion, and the primary conclusions are summarized in Section 4.

\section{Color Marker Search System}

A color marker consists of a red square and a blue square positioned side by side. The size of the marker is $100 \times$ $200 \mathrm{~mm}$, as each square measures $100 \times 100 \mathrm{~mm}$. The size of the marker was tuned to track worker behavior in our previous 
studies. In this study, the colored areas are painted blue and red on a white corrugated plastic sheet measuring $120 \times 220 \times$ $4 \mathrm{~mm}$. The width and length of the plastic plate are $20 \mathrm{~mm}$ larger than those of the painted area because the plate has a wide border area by which it can be hung and mounted on a pole without intrusion into the marker areas. To observe the marker, a single PTZ camera (EVI-D100, Sony Corp., Japan) is used, and the pan, tilt, and zoom values of the PTZ camera are controlled via RS-232C communication using VISCA commands, which is a PTZ camera control protocol.

The color marker search system integrates a marker search grid generation method, a marker region extraction method using a HSV color model, two types of marker detection filters, a particle filter developed in our previous studies, a marker distance-and-direction measurement method, and a marker 3D position estimation method with a clustering algorithm and a voting algorithm. Figure 1 shows the flowchart of the color marker search system, from the marker search grid generation process through to the marker 3D position estimation process.

\section{Marker search grid generation method}

As shown in Fig. 2, to search a wide area for multiple markers, hundreds of search grids are generated in a field. As shown in Fig. 2 (a), the grids are generated by adjusting seven parameters, namely, the following: the search distance $D$ from the PTZ camera to each grid; the step distance $\Delta D$ to change the search distance $D$; the pitch $d$ between one grid and an adjacent grid; the pan and tilt angles $\theta^{\text {pan }}$ and $\theta^{\text {tilt }}$ of the PTZ camera; the vertically upward distance $\Delta y_{\mathrm{up}}$ along the $y$ axis to tilt the PTZ camera upward; and the vertically downward distance $\Delta y_{\text {down }}$ to tilt the PTZ camera downward. In the parameter settings of distance $D$, the maximum distance and minimum distance are respectively expressed by $D_{\max }$ and $D_{\min }$. The focal length $f$ for each gird is controlled by adjusting the zoom value of the PTZ camera so that a marker with a width of $200 \mathrm{~mm}$ in an image can be captured at a preset value $h_{\text {preset }}$ in this study. The range of pan angle $\theta^{\text {pan }}$ of the PTZ camera is set from -90 to $+90^{\circ}$. Pitch $d$ between one grid and an adjacent grid is determined by considering the horizontal range of the field of view (FOV) so that the image for each grid can be overlapped on the neighboring image for the adjacent grid. The FOV can be calculated by using the distance from the camera to a grid and the camera parameters of the PTZ camera. The camera parameters were calibrated using the Tsai camera calibration method (Tsai, 1987) in our previous studies. In the $x z$-plane, the number of grids $N_{\text {grid }}(D)$ along an arc with the radius of $D$ is calculated as $N_{\text {grid }}(D)=\pi D / d$. The pan angles $\theta_{k}^{\text {pan }}(D)\left(k=0,1, \ldots, N_{\text {grid }}(D)-1\right)$ for all of the grids in the $x z$-plane are calculated by changing distance $D$. Finally,

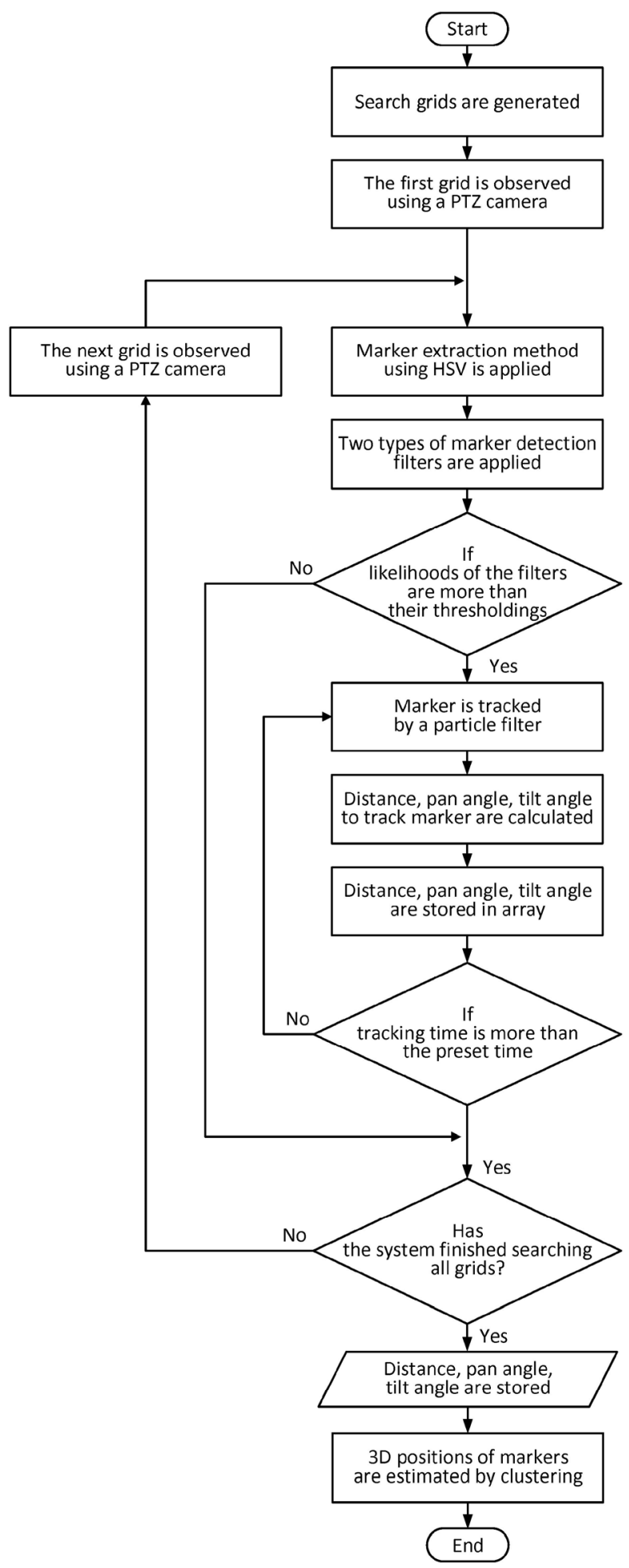

Fig. 1 Flowchart of color marker search system

as shown in Fig. 2 (b), more than 800 search grids are generated in a field when conducting a search for multiple markers.

The camera coordinates $(x, y, z)^{\mathrm{T}}$ of all of the grids in the $x z$-plane are calculated using distance $D$ and pan angle $\theta_{k}^{\text {pan }}(D)$ for each grid, as expressed by 

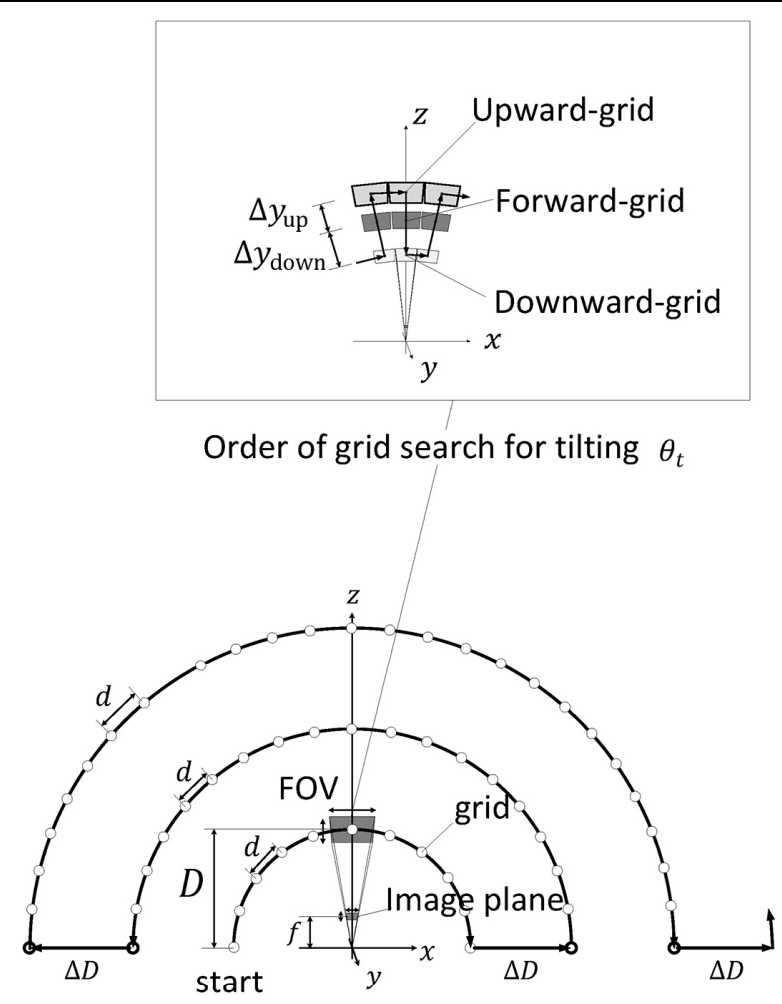

Order of grid search for panning $\theta_{p}$

(a) Generation of search grids

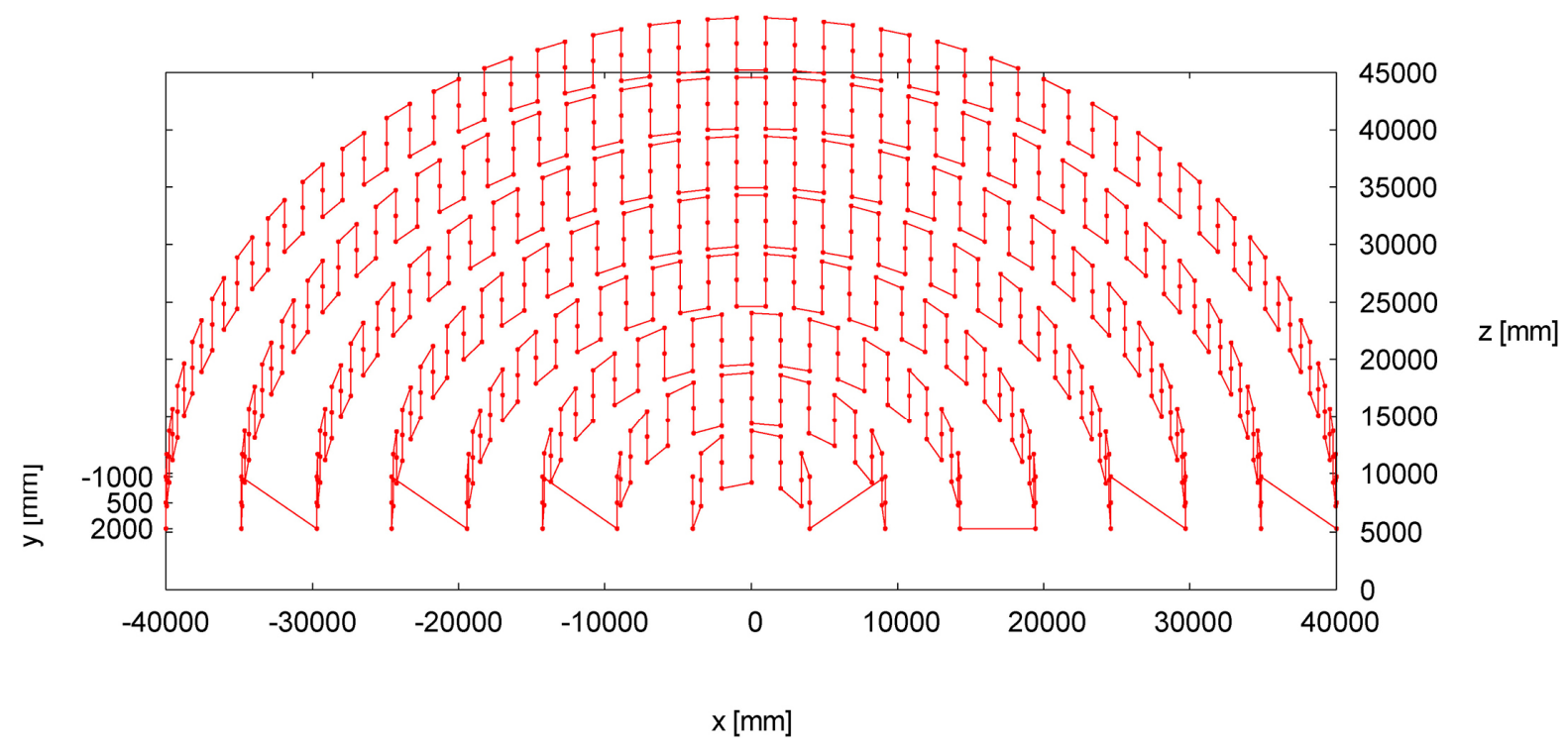

(b) Search grids ( 855 grids)

Fig. 2 Design of color marker search grids in a field and control of the pan and tilt angles of a single PTZ camera

$\left\{\begin{array}{l}x=D \sin \theta_{k}^{\mathrm{pan}}(D) \\ y=0 \\ z=D \cos \theta_{k}^{\mathrm{pan}}(D)\end{array}\right.$.

A grid on the $x z$-plane is called a forward-grid in this paper. To search for markers upward and downward as well as forward, a vertically upward grid and a vertically downward grid for each forward-grid are generated, which are respecttively called an upward-grid and a downward-grid in this study. The camera coordinates of the upward-grid are calculated by shifting the $y$-coordinate by $-\Delta y_{\text {up }}$, as expressed by $\left(D \sin \theta_{k}^{\text {pan }}(D),-\Delta y_{\text {up }}, D \cos \theta_{k}^{\text {pan }}(D)\right)$. The camera coordi- 
nates of a downward-grid are also calculated by shifting the $y$-coordinate by $+\Delta y_{\text {down }}$, as expressed by $\left(D \sin \theta_{k}^{\text {pan }}(D)\right.$, $\left.\Delta y_{\text {down }}, D \cos \theta_{k}^{\text {pan }}(D)\right)$. The upward shift distance $-\Delta y_{\text {up }}$ and the downward shift distance $+\Delta y_{\text {down }}$ are adjusted by considering the position of the camera above the ground and the vertical range of the FOV of the camera. The camera coordinates of the forward-grid are generated again with the center between the upward-grid and the downward-grid, as expressed by $\left(D \sin \theta_{k}^{\text {pan }}(D),\left(-\Delta y_{\text {up }}+\Delta y_{\text {down }}\right) / 2, D \cos \theta_{k}^{\text {pan }}(D)\right)$. Three types of grids, namely, an upward-grid, a forward-grid, and a downward-grid, are generated for a single forward-grid

As shown in Fig. 1 and 2, to start searching for markers in the field, the pan and tilt angles of the PTZ camera are first controlled to capture the first grid centered in an image. The first grid is an upward-grid having the following parameters: $D=D_{\min }, \theta_{k}^{\mathrm{pan}}(D)=-90^{\circ}$, and $y=-\Delta y_{\text {up }}$. If at least one marker is detected in the first grid, the marker is tracked and kept in the center of the image for several seconds $t_{\text {duration }}$ by applying the particle filter and controlling the pan and tilt angles of the PTZ camera. When the duration time $t_{\text {duration }}$ for tracking the marker is set to be longer, the search time to complete observing all of the grids becomes longer. The particle filter was developed in our previous studies to track in real time the center of a marker. In the particle filter, the marker area is detected by using 1,000 particles. The weight of each particle is defined so that the weight can be maximized around the boundary area between the red area and the blue area on the marker. While the marker is being tracked by the particle filter, the focal length of the PTZ camera is not changed. As shown in Fig. 1, the particle filter is applied as soon as a marker is detected by the two types of marker detection filters described in the following subsection. When a marker is not captured in an input image, the particle filter is not applied in this study.

If no marker is detected, the pan and tilt angles of the PTZ camera will be controlled to observe the second grid (Fig. 1). The second grid below the first grid is observed by tilting the PTZ camera downward, and the third grid below the second grid is likewise observed by tilting the PTZ camera downward. The first three grids are observed by tilting the PTZ camera downward from the upward-grid to the downward-grid. After observing the third grid, to observe the fourth grid, the pan angle is panned, maintaining the tilt angle. The fifth grid and the sixth grid are observed by tilting the PTZ camera upward. Whenever at least one marker is detected in each grid by the particle filter, the marker is tracked for duration time $t_{\text {duration }}$ by controlling the pan and tilt angles of the PTZ camera.

By expressing the upward-grid observation with the abbreviation $U$, a forward-grid observation with the abbreviation $F$, a downward-grid observation with the abbreviation $\mathrm{D}$, a pan rotation with the abbreviation $\mathrm{P}$, and the zoom with the abbreviation $Z$, the PTZ camera controlling sequence to observe each grid is expressed by the sequence: (UFD)-P-(DFU)-P(UFD)-P-(DFU)-P-...-(UFD)-Z-(UFD)-P-(DFU)-. When all of the grids along an arc are observed, the focal length of the PTZ camera is changed to observe the next arc without changing the pan angle or the tilt angle. Although another controlling sequence of an upward-grid search firstly, a forward-grid search secondly, and downward-grid finally as expressed by (U)-P-P-P-...-P-(F)-P-P-P-..-P-(D)-P-P-P- .. -P- were tested in the preliminary experiments, the smaller searching range for tilt angle than the final sequence pattern caused a lower score of marker detection performance. The parameters to generate the grids for searching marker are shown in Table 1.

\section{Marker region extraction method using an HSV color model}

The blue and red colors of the marker and the white color of the working uniform bearing the marker are modeled by a hue-saturation-value color model (HSV color model). To robustly extract the blue region and the red region of the marker, the three 3D HSV color histograms for blue, red, and white are trained using multiple images of the marker and the working uniform, which are taken under various lighting conditions with different color temperatures. Not only the blue histogram and the red histogram but also the white histogram is used to extract the blue and red regions of the marker because the color shift phenomena whereby the color of the white region under a cloudy sky shifts to blue and the color of the white region under an evening sky shifts to red makes it difficult to distinguish the colored marker regions from the white region. The parameter ranges of the HSV color model are defined so that the hue range is from 0 to $360^{\circ}$, the saturation range is from 0 to 1 , and the value range is from 0 to 1 . As the widths of the class interval to calculate the HSV color histograms are designed so that the width for hue is $1^{\circ}$, the width of saturation is 0.1 , and the width for value is 0.1 , the number of class intervals for hue is 360 , the number for saturation is 10 , and the number for value is 10 .

The frequencies of the 3D HSV color histograms are expressed by $M_{\text {blue }}(h, s, v)$ for blue, $M_{\text {red }}(h, s, v)$ for red, and $M_{\text {white }}(h, s, v)$ for white, where $h, s$, and $v$ are the histogram classes of hue, saturation, and value. A blue pixel or a red pixel in an input image is identified by calculating the maximum frequency $M_{\max }$ of the three color histograms $M_{\text {blue }}(h, s, v)$, $M_{\text {red }}(h, s, v)$, and $M_{\text {white }}(h, s, v)$ of an input pixel and applying thresholding to the maximum frequency, as expressed by

$M_{\text {max }}=\max \left\{M_{\text {blue }}(h, s, v), M_{\text {red }}(h, s, v), M_{\text {white }}(h, s, v)\right\}$, 
Table 1 Parameters in marker search grid generation method

\begin{tabular}{|c|c|c|}
\hline Name & Parameter & Value \\
\hline Search distance from PTZ camera to each grid [mm] & $D$ & \\
\hline Minimum search distance $[\mathrm{mm}]$ & $D_{\min }$ & 4,000 \\
\hline Maximum search distance $[\mathrm{mm}]$ & $D_{\max }$ & 40,000 \\
\hline Step distance to change search distance $[\mathrm{mm}]$ & $\Delta D$ & 5,000 \\
\hline Pitch of grid $[\mathrm{mm}]$ & $d$ & 2,000 \\
\hline Pan angle of PTZ camera $\left[^{\circ}\right]$ & $\theta^{\text {pan }}$ & -90 to +90 \\
\hline Tilt angle of PTZ camera $\left[^{\circ}\right]$ & $\theta^{\text {tilt }}$ & \\
\hline Vertically upward distance to tilt PTZ camera upward [mm] & $\Delta y_{\text {up }}$ & $-1,000$ \\
\hline Vertically downward distance to tilt PTZ camera downward [mm] & $\Delta y_{\text {down }}$ & $+2,000$ \\
\hline Focal length of PTZ camera $[\mathrm{mm}]$ & $f$ & \\
\hline Preset marker width in image [pixel] & $h_{\text {preset }}$ & 60 \\
\hline Number of grids along an arc with radius of $D$ & $N_{\text {grid }}(D)$ & \\
\hline Pan angle for the $k$-th grid along arc with radius of $D\left[^{\circ}\right]$ & $\theta_{k}^{\text {pan }}(D)$ & \\
\hline Duration time for tracking marker $[\mathrm{s}]$ & $t_{\text {duration }}$ & 1 \\
\hline Number of particles in particle filters & & 1,000 \\
\hline
\end{tabular}

Table 2 Parameters in marker region extraction method using HSV and two types of marker detection filters

\begin{tabular}{lcc}
\hline \multicolumn{1}{c}{ Name } & Parameter & Value \\
\hline Parameters in marker region extraction method using HSV & \\
Frequency of HSV color histogram for blue & $M_{\mathrm{blue}}(h, s, v)$ \\
Frequency of HSV color histogram for red & $M_{\mathrm{red}}(h, s, v)$ \\
Frequency of HSV color histogram for white & $M_{\mathrm{white}}(h, s, v)$ \\
Maximum frequency of color histograms & $M_{\mathrm{max}}$ \\
Thresholding value for maximum frequency of color histograms & $T h_{\mathrm{hsv}}$ & 10 \\
Output image by applying thresholding to maximum frequency & $I_{h s v}(i, j)$ \\
Parameters in two types of marker detection filters & \\
Side length of square window of the first filter [pixel] & $w_{1}$ \\
Likelihood of the first filter & $\pi_{1}$ & 16 \\
Thresholding value for likelihood of the first filter & $T h_{\mathrm{w}_{1}}$ \\
Output image by applying thresholding to likelihood of the first filter & $O_{w_{1}}(i, j)$ \\
Likelihood for the $k$-th posture type in candidate area of $l$ & $\pi_{2}^{k}(l)$ \\
Side length of square window of the second filter [pixel] & $w_{2}$ \\
Maximum likelihood of the second filter in candidate area of $l$ & $\pi_{2}^{\text {max }}(l)$ \\
Maximum likelihood of the second filter over all candidate areas & $\hat{\pi}_{2}^{l}$ \\
Thresholding value for maximum likelihood of the second filter & $T h_{\mathrm{w}_{2}}$ \\
Binary image output by applying thresholding to likelihood of the second filter & $O_{w_{2}}$ \\
Likelihood of the first filter at the $k$-th pixel in marker area & $\pi_{1, k}$ \\
Image coordinates of the $k$-th pixel in the candidate area & $\boldsymbol{x}_{k}$ \\
Marker center position estimated by a weighted mean & $\overline{\boldsymbol{x}}$ \\
\hline
\end{tabular}

$I_{h s v}(i, j)=\left\{\begin{array}{cl}\text { blue } & \text { if } M_{\max }=M_{\mathrm{blue}}(h, s, v) \text { and } M_{\mathrm{max}} \geq T h_{\mathrm{hsv}} \\ \text { red } & \text { if } M_{\max }=M_{\mathrm{red}}(h, s, v) \text { and } M_{\max } \geq T h_{\mathrm{hsv}} \\ 0 & \text { else }\end{array}\right.$

where $I_{h s v}(i, j)$ is the output at the image coordinate $(i, j)$, and $T h_{\mathrm{hsv}}$ is the thresholding value for the maximum frequency $M_{\max }$. For example, when the color with the maximum frequency is blue and the frequency is higher than the thresholding value, the pixel is identified as a blue pixel. When the maximum frequency is smaller than the thresholding value, the pixel is identified as a background pixel. The parameters to extract marker region using HSV color histogram are shown in Table 2. 


\section{Two types of marker detection filters}

As an image having a VGA resolution is continuously being captured without stopping control of the pan and tilt angles, the input image is a little blurred. To robustly and quickly detect a marker in the input image, two types of marker detection filters are used for applying a coarse-to-fine approach, as shown in Fig. 3. First, as shown in Fig. 3 (a), the first filter extracts pixels around the boundary area between the blue region and the red region of each marker in an input image. The likelihood $\pi_{1}$ of the first filter extracting a pixel

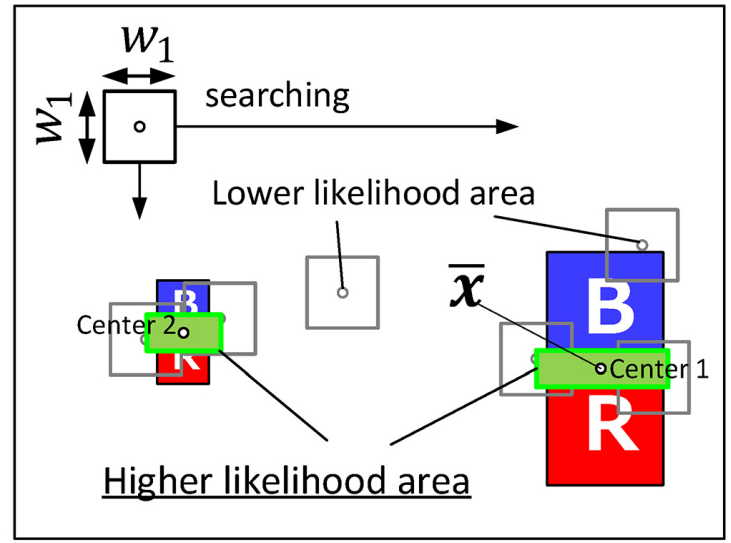

(a) The first filter detects some blue-and-red boundary areas
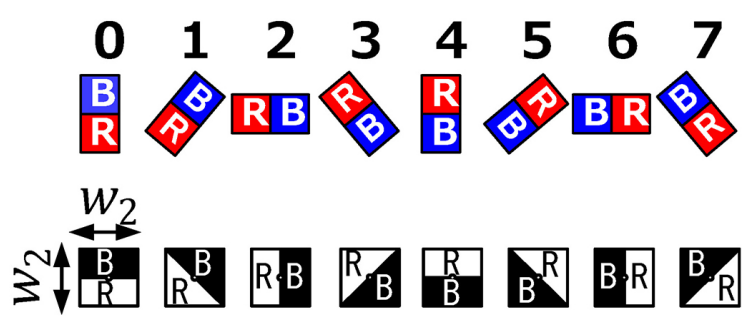

(b) The second filter determines a single color marker candidate

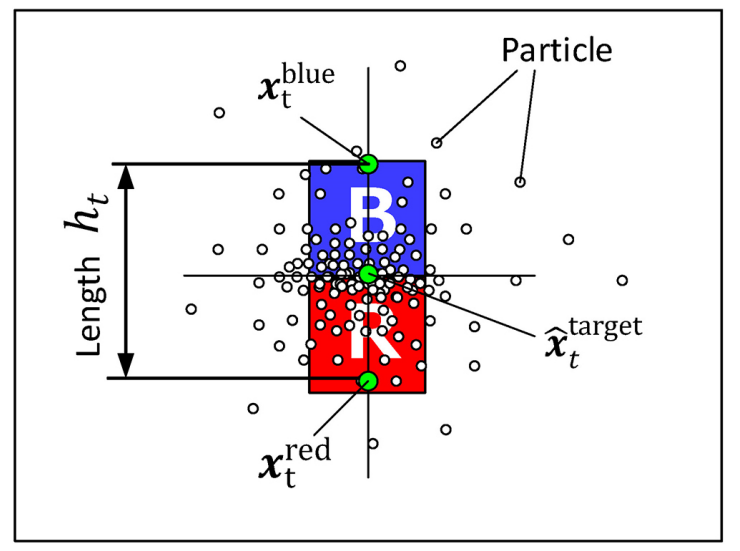

(c) Tracking of the center of a color marker by the particle filter and detection of marker length

Fig. 3 Two marker detection filters are used to detect color markers with a coarse-to-fine approach for quick marker detection around the blue-and-red boundary area is defined by

$$
\pi_{1}=\frac{n_{B} \times n_{R}}{w_{1} \times w_{1}},
$$

where $n_{B}$ and $n_{R}$ are respectively the numbers of blue pixels and red pixels in the square window around a pixel of interest, and $w_{1}$ is the side length of the window. To quickly count the number of blue and red pixels in the window, an integral image is used in the filter. The integral image was used to quickly compute human facial features by Viola and Jones (Viola and Jones, 2001). The output $O_{w_{1}}$ of the first filter for each pixel is computed by applying thresholding to likelihood $\pi_{1}$ as expressed by

$$
O_{w_{1}}(i, j)=\left\{\begin{array}{cc}
1 & \pi_{1}>T h_{\mathrm{w}_{1}} \\
0 & \text { else }
\end{array}\right.
$$

where $O_{w_{1}}(i, j)$ is the output of the first filter at the image coordinates $(i, j)$, and $T h_{\mathrm{w}_{1}}$ is the thresholding value for likelihood $\pi_{1}$. A labeling method is applied to the blue-andred boundary areas detected by the first filter, and the center position of each area is detected in the first filter. The center positions of the labeled areas are referred as the candidates of the color markers.

Second, to determine whether a detected candidate is a marker, as shown in Fig.3 (b), a second filter is employed that uses all eight likelihoods for each of the eight marker posture types. The eight likelihoods $\pi_{2}^{k}(l)(k=0,1, \ldots, 7)$ at each candidate position are computed as expressed by

$$
\pi_{2}^{k}(l)=\frac{n_{B}^{k}(l) \times n_{R}^{k}(l)}{w_{2} \times w_{2}},
$$

where $k$ is the posture number of the eight marker posture types, $\pi_{2}^{k}(l)$ is the likelihood of the $k$-th posture type of the candidate area with a label number $l, n_{B}^{k}(l)$ and $n_{R}^{k}(l)$ are respectively the numbers of blue pixels and red pixels in the $k$-th square window placed at the center of the candidate area with the label number of $l$, and $w_{2}$ is the side length of the window. To precisely calculate the likelihood without the calculation being affected by the marker posture, the argument for the maximum of eight likelihoods $\pi_{2}^{\max }(l)$ for a candidate with label $l$ is computed by

$$
\pi_{2}^{\max }(l)=\arg \max _{0 \leq k \leq 7} \pi_{2}^{k}(l) .
$$

Third, the number of marker candidates is reduced to one by computing $\hat{\pi}_{2}^{\hat{l}}$, the argument of the maximum of the $\pi_{2}^{\max }(l)$ over all candidates, as expressed by

$$
\hat{\pi}_{2}^{\hat{l}}=\arg \max _{0 \leq l \leq L} \pi_{2}^{\max }(l),
$$

where $\hat{l}$ is the label number of the candidate area with the maximum $\hat{\pi}_{2}^{\hat{l}}$, and $L$ is the total number of label numbers of the candidate areas. The output $O_{w_{2}}(i, j)$ of the second filter 
for each pixel in the candidate area the label number of $\hat{l}$ is computed by applying thresholding to likelihood $\hat{\pi}_{2}^{\hat{l}}$. If likelihood $\hat{\pi}_{2}^{\hat{l}}$ is more than thresholding value $T h_{\mathrm{w}_{2}}$, all of the pixels in the candidate area are detected as a targeted pixel to be tracked with the PTZ camera.

As the image captured by the PTZ camera is a little blurred, the center position of the detected marker candidate area is shifted a little from the true position. Finally, to more precisely detect the center position of the marker, the center position $\bar{x}$ is estimated by calculating a weighted mean, as expressed by

$$
\overline{\boldsymbol{x}}=\frac{\sum_{k=1}^{M} \pi_{1, k} \boldsymbol{x}_{k}}{\sum_{k=1}^{M} \pi_{1, k}},
$$

where $\pi_{1, k}$ is the likelihood of the first filter at the $k$-th pixel in the candidate area with the label number of $\hat{l}, \boldsymbol{x}_{k}$ is the image coordinates of the $k$-th pixel in the candidate area, and $M$ is the number of pixels in the candidate area. The parameters to estimate the center position of a marker using two types of detection filters are shown in Table 2.

\section{Marker distance-and-direction measurement method}

To measure the distance from the camera to a marker and the direction of the marker to the PTZ camera, the following three steps are applied in this study:

First, as soon as final marker candidate $\overline{\boldsymbol{x}}$ has been detected, the pan and tilt angles are controlled so that candidate $\overline{\boldsymbol{x}}$ is captured and centered in the input image; then, the processing mode of the system is changed from marker search mode to marker tracking mode.

Second, as shown in Fig. 3 (c), for several seconds $t_{\text {duration }}$, the center of the marker continues to be detected by the particle filter and captured in the center of the image plane by controlling the pan and tilt angles. In the $t$-th frame in tracking mode using the particle filter, the center coordinates of the marker $\widehat{\boldsymbol{x}}_{t}^{\text {target }}$ in the input image and the absolute pan and tilt angles $\theta_{t}^{\text {pan }}$ and $\theta_{t}^{\text {tilt }}$ used to capture the marker centered in the image are computed as the parameters to calculate the marker's $3 \mathrm{D}$ position.

Third, the length $h_{t}[\mathrm{~mm}]$ of the marker in the image plane in the $t$-th frame is measured by using the edge point $\boldsymbol{x}_{\mathrm{t}}^{\text {blue }}$ of the blue marker and the edge point $\boldsymbol{x}_{t}^{\text {red }}$ of the red marker around the center $\widehat{\boldsymbol{x}}_{t}^{\mathrm{target}}$, as expressed by $h_{t}=$ $\left|\boldsymbol{x}_{t}^{\mathrm{blue}}-\boldsymbol{x}_{t}^{\mathrm{red}}\right|$. The edge points of $\boldsymbol{x}_{\mathrm{t}}^{\mathrm{blue}}$ and $\boldsymbol{x}_{t}^{\mathrm{red}}$ are detected by searching the nearest blue-labeled-area and red-labeled-area to the center coordinates of the marker $\widehat{\boldsymbol{x}}_{t}^{\text {target }}$. After finding the nearest blue-labeled-area and red-labeled-area, two straight lines from the center coordinates of the marker $\widehat{\boldsymbol{x}}_{t}^{\mathrm{target}}$ to the center coordinates of the blue-labeled-area and the red-labeled-area are independently drawn to search not only a pixel at the edge of blue-labeled-area but also a pixel at the edge of the red-labeled-area. Finally, the pixel at the edge of the blue-labeled-area and the pixel at the edge of the red-labeled-area are detected as by independently projecting all of pixels on the blue-labeled-area and the red-labeled-area onto the two straight lines and searching a pixel with the maximum distance from the center coordinates of the marker $\widehat{x}_{t}^{\text {target }}$ along each straight line. The actual coordinates of the edge points of $\boldsymbol{x}_{\mathrm{t}}^{\mathrm{blue}}[\mathrm{mm}]$ and $\boldsymbol{x}_{t}^{\text {red }}[\mathrm{mm}]$ without lens distortion in the image plane are calculated by using the camera parameters estimated by Tsai camera calibration method. The length $h_{t}$ of the marker is measured without dependence on marker postures.

The distance $D_{t}$ from the camera to the marker in the $t$-th frame is calculated by applying triangulation with length $h_{t}$ of the marker in the image, the actual length $H(200 \mathrm{~mm})$ of the marker, and the focal length $f_{t}$ in the $t$-th frame, as expressed by

$$
D_{t}=f_{t} \frac{H}{h_{t}} .
$$

While the marker is being tracked by the particle filter for duration time $t_{\text {duration, }}$, the datasets of distance, and the pan and tilt angles $\left(D_{t}, \theta_{t}^{\text {pan }}, \theta_{t}^{\text {tilt }}\right)(t=0, \ldots, N-1)$ continue to be stored in a buffer memory at a frame rate of about 30 frames / $\mathrm{s}$ in order to measure the 3D position of the marker, where $N$ is the total number of datasets. As multiple markers are tracked simultaneously, the $N$ datasets $\left(D_{t}, \theta_{t}^{\text {pan }}, \theta_{t}^{\text {tilt }}\right)$ include the measured values of some markers without classifying the markers. The parameters to measure the distance-and-direction of a marker are shown in Table 3.

\section{Estimating the 3D position of a marker using a clustering algorithm}

Because the datasets $\left(D_{t}, \theta_{t}^{\text {pan }}, \theta_{t}^{\text {tilt }}\right)(t=0, \ldots, N-1)$ stored in the buffer memory are not classified into classes to distinguish multiple markers, as shown in Fig. 4 (a), the nearest neighbor clustering method is applied to classify the datasets into a cluster $\left({ }^{c} D_{s},{ }^{c} \theta_{s}^{\text {pan }},{ }^{c} \theta_{s}^{\text {tilt }}\right)\left(c=0, \ldots, N_{c}-\right.$ $\left.1, s=0, \ldots, N_{s}-1\right)$ for each marker in order to separately measure its $3 \mathrm{D}$ position, where ${ }^{c} D_{s},{ }^{c} \theta_{s}^{\text {pan }}$, and ${ }^{c} \theta_{s}^{\text {tilt }}$ are respectively the distance, pan angle, and tilt angle of the $s$-th sample classified into the cluster $c, N_{c}$ is the number of clusters, and $N_{S}$ is the number of samples in the cluster $c$. The nearest neighbor method uses 2D Euclidean distance in the pan and tilt angle space $\left(\theta_{t}^{\mathrm{pan}}, \theta_{t}^{\text {tilt }}\right)$ to classify the datasets. The thresholding value $T h_{N N}$ for the Euclidean distance for clustering is tuned in preliminary experiments.

The true distance ${ }^{c} \widehat{D}$ of cluster $c$ is estimated by using the voting method shown in Fig. 4 (b). For voting, the 
Table 3 Parameters in measuring marker distance-and-direction and estimating marker 3D position

\begin{tabular}{|c|c|c|}
\hline Name & Parameter & Value \\
\hline \multicolumn{3}{|l|}{ Parameters to measure marker distance-and-direction } \\
\hline Center coordinates of targeted marker at the $t$-th frame & $\widehat{\boldsymbol{x}}_{t}^{\mathrm{target}}$ & \\
\hline Absolute pan angle to capture marker centered in image at the $t$-th frame $\left[{ }^{\circ}\right]$ & $\theta_{t}^{\mathrm{pan}}$ & \\
\hline Absolute tilt angle to capture marker centered in image at the $t$-th frame $\left[{ }^{\circ}\right]$ & $\theta_{t}^{\text {tilt }}$ & \\
\hline Length of the marker in the image plane at the $t$-th frame $[\mathrm{mm}]$ & $h_{t}$ & \\
\hline Actual length of the marker $[\mathrm{mm}]$ & $H$ & 200 \\
\hline Focal length at the $t$-th frame $[\mathrm{mm}]$ & $f_{t}$ & \\
\hline Distance from camera to marker at the $t$-th frame & $D_{t}$ & \\
\hline \multicolumn{3}{|l|}{ Parameters to estimate 3D position of marker using clustering algorithm } \\
\hline Number of clusters in nearest neighbor clustering & $N_{c}$ & \\
\hline Number of samples in cluster $c$ & $N_{s}$ & \\
\hline Distance of the $s$-th sample classified into the cluster $c[\mathrm{~mm}]$ & ${ }^{c} D_{S}$ & \\
\hline Pan angle of the $s$-th sample classified into the cluster $c\left[^{\circ}\right]$ & ${ }^{c} \theta_{s}^{\text {pan }}$ & \\
\hline Tilt angle of the $s$-th sample classified into the cluster $c\left[^{\circ}\right]$ & ${ }^{c} \theta_{S}^{\text {tilt }}$ & \\
\hline Thresholding value for Euclidean distance in nearest neighbor clustering $\left[^{\circ}\right]$ & $T h_{\mathrm{NN}}$ & 2 \\
\hline True distance of cluster $c$ estimated by voting method [mm] & ${ }^{c} \widehat{D}$ & \\
\hline Number of levels in distance parameter space & $N_{D}$ & \\
\hline Pitch distance of distance parameter space $[\mathrm{mm}]$ & $p$ & 100 \\
\hline Maximum distance in voting method $[\mathrm{mm}]$ & $D_{\max }^{\text {vote }}$ & 50,000 \\
\hline Voting range around the distance of ${ }^{c} D_{s}$ in cluster $c[\mathrm{~mm}]$ & $D_{\text {range }}^{\text {vote }}$ & 1,000 \\
\hline Thresholding value for the maximum frequency of the voting array & $T h_{\text {vote }}$ & 15 \\
\hline Average pan angle of the $c$-th cluster $\left[{ }^{\circ}\right]$ & ${ }^{c} \hat{\theta}^{\text {pan }}$ & \\
\hline Average tilt angle of the $c$-th cluster $\left[{ }^{\circ}\right]$ & ${ }^{c} \hat{\theta}^{\text {tilt }}$ & \\
\hline Camera coordinates of the $c$-th cluster as actual marker position & $\left({ }^{c} x,{ }^{c} y,{ }^{c} z\right)$ & \\
\hline ENU coordinates of the $c$-th cluster as actual marker position & $\left({ }^{c} E,{ }^{c} N,{ }^{c} U\right)$ & \\
\hline
\end{tabular}

parameter space of distance $D$ from the maximum distance $D_{\max }^{\text {vote }}$ to 0 is divided into $N_{D}$ levels with pitch $p$. Voting is performed for each cluster by using $N_{S}$ sets of distances ${ }^{c} D_{s}\left(s=0, \ldots, N_{s}-1\right)$. As shown in Fig. 4 (b), in voting for the $s$-th distance ${ }^{c} D_{s}$ in the cluster $c$, within the distance range from the lower limit $\left({ }^{c} D_{s}-D_{\text {range }}^{\text {vote }}\right)$ to the upper limit $\left({ }^{c} D_{s}+D_{\text {range }}^{\text {vote }}\right)$ around the distance ${ }^{c} D_{s}$, the values of all of the bins from $d_{i-m}$ to $d_{i+m}$ around $d_{i}$ in a one-dimensional accumulator array are incremented, where $D_{\text {range }}^{\text {vote }}$ is the voting range around measured distance ${ }^{c} D_{s}, d_{i}$ is the $i$-th bin position in the voting array corresponding to the distance of ${ }^{c} D_{S}$, and $m$ is the number of the bins in the voting array corresponding to the distance of $D_{\text {range }}^{\text {vote }}$ The true distance ${ }^{c} \widehat{D}$ of each cluster is estimated by both detecting the maximum frequency of the voting array and applying thresholding to the maximum frequency using thresholding value $T h_{\text {vote }}$.

The 3D position $\left({ }^{c} x,{ }^{c} y,{ }^{c} z\right)$ of the $c$-th marker classified by the nearest neighbor method is computed by

$$
\left\{\begin{array}{l}
{ }^{c} x={ }^{c} \widehat{D} \cos { }^{c} \hat{\theta}^{\mathrm{tilt}} \sin { }^{c} \hat{\theta}^{\mathrm{pan}} \\
{ }^{c} y=-{ }^{c} \widehat{D} \sin { }^{c} \hat{\theta}^{\mathrm{tilt}} \\
{ }^{c} z={ }^{c} \widehat{D} \cos { }^{c} \widehat{\theta}^{\mathrm{tilt}} \cos { }^{c} \hat{\theta}^{\mathrm{pan}}
\end{array},\right.
$$

where ${ }^{c} \hat{\theta}^{\text {pan }}$ and ${ }^{c} \hat{\theta}^{\text {tilt }}$ are respectively the average pan and tilt angles of the $c$-th cluster.

The actual 3D position of each marker in the ENU coordinate system is measured using the fixed solution of the RTK GNSS device. The camera coordinates $\left({ }^{c} x,{ }^{c} y,{ }^{c} z\right)^{T}$ measured by the marker search system are converted into ENU coordinates $\left({ }^{c} E,{ }^{c} N,{ }^{c} U\right)^{T}$, as expressed by

$$
\left[\begin{array}{c}
{ }^{c} E \\
{ }^{c} N \\
{ }^{c} U
\end{array}\right]=\boldsymbol{R}\left[\begin{array}{c}
{ }^{c} x \\
{ }^{c} y \\
{ }^{c} Z
\end{array}\right]
$$

where the origin of the ENU coordinate system is located at the origin of the camera coordinate system and $\boldsymbol{R}$ is a $3 \times 3$ conversion matrix. $\boldsymbol{R}$ is estimated by the least mean square method using regression data of the ENU coordinates measured by RTK GNSS and the camera coordinates measured by the marker search system in preliminary experiments. The parameters to estimate the marker 3D position are shown in Table 3. 
1

Cluster No.

2

3

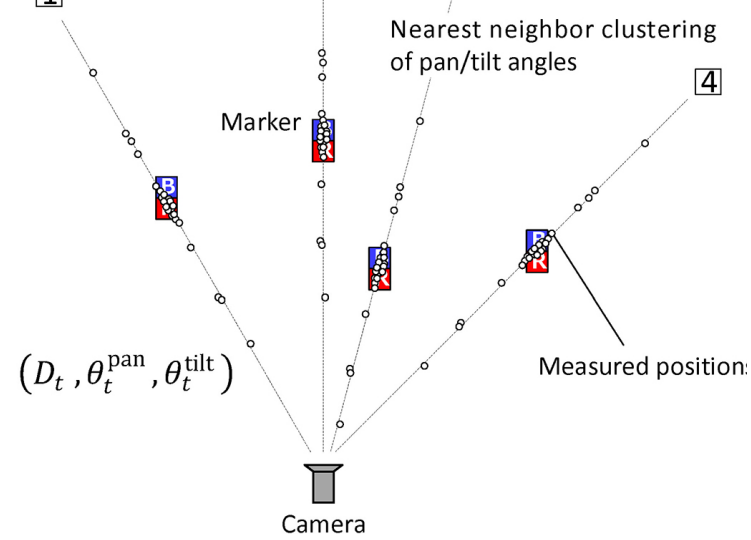

(a) Pan and tilt angle clustering by nearest neighbor clustering

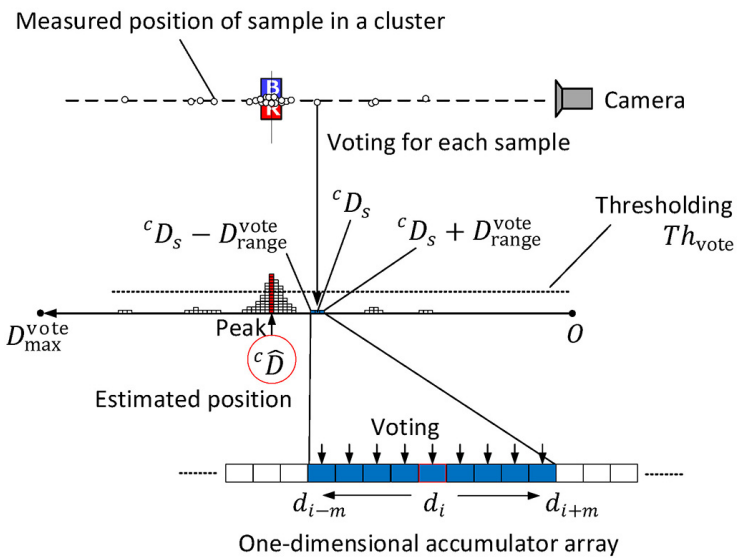

(b) Voting method used to estimate distance

Fig. 4 Nearest neighbor clustering of pan-tilt angles and voting method used to estimate the distance from camera to marker

\section{Experimentation}

To test the performance of our developed color marker search system, two sets of experiments (No. 1 and 2) were conducted using a horizontal cultivated agricultural field ( $30 \mathrm{~m}$ wide $\times 50 \mathrm{~m}$ deep). To build a prototype of the proposed marker search system, a single PTZ camera with $\mathrm{a} \times 10$ optical zoom magnification and a personal computer (CPU: Intel Core i7-2700k@3.05 GHz, OS: Windows 764 bit)with a video board (MOR / 4VD, Matrox Electronic Systems Ltd., Canada) were installed on the cargo bed of a 4-wheel electrically assisted vehicle (CB-01B, Yamaichi Seiko Co. Ltd., Japan) (length $1,400 \times$ width $780 \times$ height $880 \mathrm{~mm}$ ). The PTZ camera was positioned $1.8 \mathrm{~m}$ above the ground, and its iris and the white balance were set to auto. The digital zoom magnification of PTZ camera was not used in the experiments, because the same color of a marker as the color in training of the HSV color histogram might not be acquired in testing

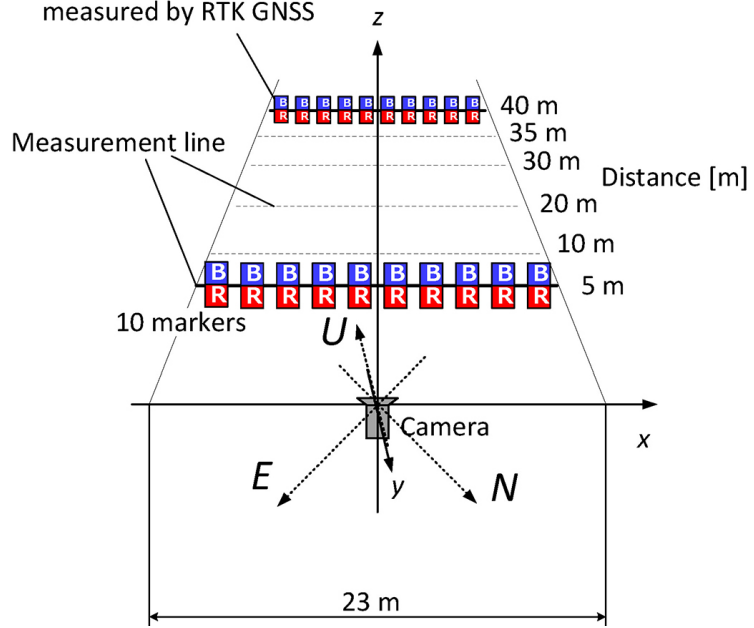

(a) Placement along 6 lines

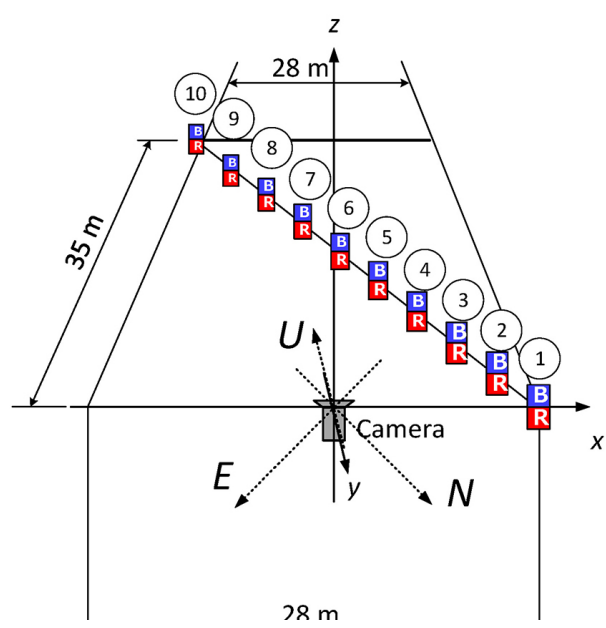

(b) Placement along diagonal line

Fig. 5 Two experiments using two patterns of marker placement and measurement of positions of 10 markers in the ENU coordinate system

when a marker was placed far from the camera. A multithreaded program with an image processing thread and a PTZ camera-controlling thread was developed in Microsoft Visual $\mathrm{C}++$. The input image size was $640 \times 480$ pixels. The $\mathrm{x}$ and $\mathrm{y}$ dimension of the PTZ camera sensor element were 0.00475 and $0.00555 \mathrm{~mm}$ respectively. The frame rate for the image processing was approximately 30 frames / s.

\section{Setup of experiment No. 1}

Experiment No. 1 was conducted on three dates (19th, 20th, and 28th Jan. 2015). To test the precision of the measurement of the 3D positions of markers, as shown in Fig. 5 (a), 10 markers were placed facing to the PTZ camera along a measurement line with a length of approximately $23 \mathrm{~m}$. As the 10 markers were roughly divided into 5 and 5 at practically equal distances from each other, the distance between adjacent markers along the line was approximately $2.5 \mathrm{~m}$. In 
the experiments, the $3 \mathrm{D}$ positions of the 10 markers were measured by changing the distance from the camera to the measurement line between 6 distances: 5, 10, 20,30, 35, and $40 \mathrm{~m}$ (Fig. 5 (a)). In each experiment, 10 markers were placed along each measurement line. Each marker was fixed and mounted on a polyvinyl chloride pipe at a point $1.5 \mathrm{~m}$ above the ground so that the blue and red areas of the marker were ordered vertically as shown in Fig. 5. As the height differences between the positions of all of the markers and the origin of the camera coordinates had a small range, approximately $-97 \mathrm{~mm}$ to $+130 \mathrm{~mm}$, the height of any one of the markers was almost the same as that of the camera. The actual $3 \mathrm{D}$ position of each marker in the ENU coordinate system was measured using a fixed RTK solution from a RTK GNSS device (A325, Hemisphere GNSS Inc., USA).

On the first day, to test the performance of the marker search system in an area near the camera, 10 markers were placed along the line position at $10 \mathrm{~m}$. Unfortunately, diagonally to the left and to the rear the field, the red steel frames of a building under construction covered with blue protection nets were captured in the background when the camera was panned to capture the grids around the edge of the left side of the field. The blue and red construction materials were extracted as image noise, which made it difficult to detect markers. On the second day, to ascertain the upper limit of the search distance, 10 markers were placed along four positions on the measurement line by changing the line position between distances greater than $10 \mathrm{~m}$, that is, $20,30,35$, and $40 \mathrm{~m}$. On the third day, 10 markers were placed along a line position of $5 \mathrm{~m}$ to demonstrate again the performance of the marker search system in the area close to the camera under the lighting conditions of another day. Throughout the three days, as strong winds occasionally blew, some markers swayed in the wind during the search. As the sky was not clear at all times (it was sometimes cloudy and occasionally it snowed a little), the lighting conditions for capturing images changed throughout the three days. By means of the six settings of line positions, each marker was captured separately in an image without overlapping with other markers.

\section{Setup of experiment No. 2}

To test the robustness of the marker search system all day long, experiment No. 2 was conducted in the same field as experiment No. 1 on another date (16th Apr. 2015). As shown in Fig. 5 (b), 10 markers were placed facing to the PTZ camera at equal intervals along a diagonal line setup in the experimental region $(28 \mathrm{~m}$ wide $\times 35 \mathrm{~m}$ deep $)$, and the placement of the 10 markers was fixed throughout the experiments by hanging the markers on polyvinyl chloride pipes at points $1.5 \mathrm{~m}$ above the ground so that the blue and red areas of the marker were ordered vertically as shown in Fig. 5. As the height differences between the positions of all of the markers and the origin of the camera coordinates had a small range, approximately -263 to $+40 \mathrm{~mm}$, the height of any one of the markers was almost the same as that of the camera. The actual 3D position of each marker in the ENU coordinate system was measured using a fixed RTK solution from a RTK GNSS device. In experiment No. 2, the 3D position of each marker was measured 18 times from late morning to late afternoon, namely, at the following times: 11:36, 11:43, 11:50, $12: 46,12: 53,12: 59,13: 46,13: 54,14: 01,14: 36,14: 43,14: 50$, $15: 40,15: 46,15: 53,16: 32,16: 39$, and 16:45. As for weather conditions, it was a partially cloudy until 14:30, when it became overcast, but unlike the case for experiment No. 1, there were no strong winds and the markers were not disturbed. Fortunately, the large obstacle of construction materials having colors similar to those of the markers observed in experiment No. 1 had been removed before the start of experiment No. 2. All the markers were captured separately in images without overlapping the other markers.

\section{Parameter settings}

In the parameter settings for grid generation, as shown in Table 4, distance $D$ was adjusted in eight steps of Nos. 1 to 8 from a minimum distance $D_{\min }$ of $4 \mathrm{~m}$ to a maximum distance $D_{\text {max }}$ of $40 \mathrm{~m}$. The maximum distance of $40 \mathrm{~m}$ was the upper limit of the range at which the PTZ camera could detect markers, and the minimum distance of $4 \mathrm{~m}$ was determined as the lower limit of the worker assistance zone of our system, and the step distance $\Delta D$ is approximately $5 \mathrm{~m}$. The focal length $f$ for each gird was set so that a marker with a length of $200 \mathrm{~mm}$ in an image was able to be captured at a preset value $h_{\text {preset }}$ of 60 pixels. To overlap one image in a grid onto another image in an adjacent grid, horizontal pitch $d$ between two grids was adjusted to $2 \mathrm{~m}$. To generate upward-grids,

Table 4 Eight sets of search distances, focal lengths, and numbers of grids

\begin{tabular}{ccccc}
\hline No. & $\begin{array}{c}\text { Distance } \\
{[\mathrm{mm}]}\end{array}$ & $\begin{array}{c}\text { Focal length } \\
{[\mathrm{mm}]}\end{array}$ & \multicolumn{2}{c}{$\begin{array}{c}\text { Number of grids } \\
\text { (Forward-grids) }\end{array}$} \\
\hline 1 & 4,000 & 6.7 & 21 & $(7)$ \\
2 & 9,143 & 15.3 & 45 & $(15)$ \\
3 & 14,286 & 23.8 & 69 & $(23)$ \\
4 & 19,429 & 31.1 & 96 & $(32)$ \\
5 & 24,571 & 31.1 & 120 & $(40)$ \\
6 & 29,714 & 31.1 & 144 & $(48)$ \\
7 & 34,857 & 31.1 & 168 & $(56)$ \\
8 & 40,000 & 31.1 & 192 & $(64)$ \\
\hline & & Total & 855 & $(285)$ \\
\hline
\end{tabular}


forward-grids, and downward-grids, the upward shift distance $-\Delta y_{\text {up }}$ was adjusted to $-1,000 \mathrm{~mm}$, and the downward shift distance $+\Delta y_{\text {down }}$ was adjusted to $+2,000 \mathrm{~mm}$. As the three types of grids - an upward-grid, a forward-grid, and a downward-grid - were generated for a single forward-grid, the total number of grids became 3 times as many as the total number of forward-grids, as shown in Table 4 . As the number of forward-grids was 285 , the total number of grids was 855 . Finally, 855 search grids were generated in the field (Fig. 2 (b)).

In the parameter settings of the two types of marker detection filters, the window size of the first filter $w_{1}$ was set to 16 pixels and the thresholding value $T h_{\mathrm{w}_{1}}$ for the likelihood $\pi_{1}$ was set to 1.0. For the second filter, the window size $w_{2}$ was set to 30 pixels and the thresholding value $T h_{\mathrm{w}_{2}}$ for the likelihood $\hat{\pi}_{2}^{\hat{l}}$ was set to 1.0. The parameters of the window sizes of $w_{1}$ and $w_{2}$ and the thresholding values of $T h_{\mathrm{w}_{1}}$ and $T h_{\mathrm{w}_{2}}$ were the key parameters to detect marker area in an image. The parameters were tuned in the preliminary experiments so that the marker area was able to be detected robustly preventing misdetections of the non-marker areas. In the parameter settings of the particle filter used for tracking the markers, the duration time $t_{\text {duration }}$ for tracking a marker was set to $1 \mathrm{~s}$.

To smoothly and as quickly as possible control the pan angle, the tilt angle, and the zoom value of the PTZ camera, the time interval to send a VISCA command to the PTZ camera was tuned by considering the time required to control the pan and tilt action of the PTZ camera (pan speed: $300^{\circ} / \mathrm{s}$, tilt speed: $125^{\circ} / \mathrm{s}$ ) and the image processing frame rate (about 30 frames / s) so that the marker was able to be detected robustly even if the image captured by the camera was a little blurred.

In the parameter settings of the three 3D HSV color histograms used to extract the marker region, the frequencies of the three histograms $M_{\text {blue }}, M_{\text {red }}$, and $M_{\text {white }}$ were counted using images taken under fluorescent light in a room, a blue clear sky, a cloudy sky, noon sunlight, average daylight, and evening sunlight in preliminary experiments. Multiple samples of the blue and red areas of the marker, and the white area of the working uniform were trimmed manually to count the frequency of each histogram. In this study, the number of training samples was 79,192 pixels for blue, 626,668 pixels for red, and 54,982 pixels for white. The frequencies of the three histograms were not normalized, and the thresholding values $T h_{\mathrm{hsv}}$ for the three frequencies were set to the same value of 10 across the three histograms.

In the parameter settings for clustering markers using the nearest neighbor method, the thresholding value $T h_{\mathrm{NN}}$ for the 2D Euclidean distance in the pan and tilt angle space $\left(\theta_{t}^{\text {pan }}, \theta_{t}^{\text {tilt }}\right)$ was adjusted to $2^{\circ}$. In the parameter settings of the voting method for estimating the $3 \mathrm{D}$ positions of multiple markers, as the pitch and the maximum distance were set so that $p=100 \mathrm{~mm}$ and $D_{\max }^{\text {vote }}=50,000 \mathrm{~mm}$, the number of distance levels $N_{D}$ was set to 500 . Moreover, as the voting range $D_{\text {range }}^{\text {vote }}$ was tuned to $1,000 \mathrm{~mm}$ and the voting pitch $p$ was set to $100 \mathrm{~mm}$, the voting bin range $m$ was set to 10 . The voting pitch $p$ corresponding to the resolution for measuring distance was $100 \mathrm{~mm}$. The thresholding value $T h_{\text {vote }}$ for the maximum frequency of the voted array was set to 15 .

The conversion matrix $\boldsymbol{R}$ from the camera coordinates to the ENU coordinates was estimated using regression data, with the actual marker positions measured by the RTK GNSS device and the camera coordinates measured precisely by the marker search system. The errors of the conversion from the camera coordinate to ENU coordinates in the two experiments were that the root mean square error (RMSE) in experiment No. 1 was 0.39 m, and No. 2 was $0.33 \mathrm{~m}$.

\section{Results and Discussion}

\section{Results of experiment No. 1}

Figure 6 (a) shows the results for the measurements of the 3D positions of the 10 markers placed along the six line positions of 5, 10, 20,30,35, and $40 \mathrm{~m}$ in the ENU coordinate system. The detection rates for the 5 and $10 \mathrm{~m}$ line positions were $100 \%(10 / 10)$, the rate for $20 \mathrm{~m}$ was $90 \%(9 / 10)$, the rate for $30 \mathrm{~m}$ was $80 \%(8 / 10)$, the rate for $35 \mathrm{~m}$ was $40 \%$ (4 $/ 10)$, and the rate for $40 \mathrm{~m}$ was $0 \%(0 / 10)$. When the measurement line was placed within $30 \mathrm{~m}$, almost all the 3D positions of the markers could be measured consistently. However, within $30 \mathrm{~m}$, one marker for the $20 \mathrm{~m}$ line position and two markers for the $30 \mathrm{~m}$ line position were not detected because the markers could not be distinguished from the red and blue construction materials in the background. When the measurement line was placed at a position greater than $30 \mathrm{~m}$, for example, 35 or $40 \mathrm{~m}$, almost none of the markers could be detected consistently because the search distance from the camera was too far to robustly detect them. The results indicate that the upper limit of the marker placement line position was approximately $30 \mathrm{~m}$. As the maximum distance of the 10 markers placed at the line position of $30 \mathrm{~m}$ was $32.5 \mathrm{~m}$, the upper limit of the sensing range was considered to be approximately $32.5 \mathrm{~m}$. Within the sensing range of $32.5 \mathrm{~m}$, the detection rate of the 40 positions was calculated as $92.5 \%$ $(37 / 40)$.

Figure 6 (b) shows the measurement error histogram of the 10 markers placed along the five line positions of $5,10,20$, 30 , and $35 \mathrm{~m}$. When the distance from the camera to the line position was small, for example, 5 and $10 \mathrm{~m}$, most measure- 


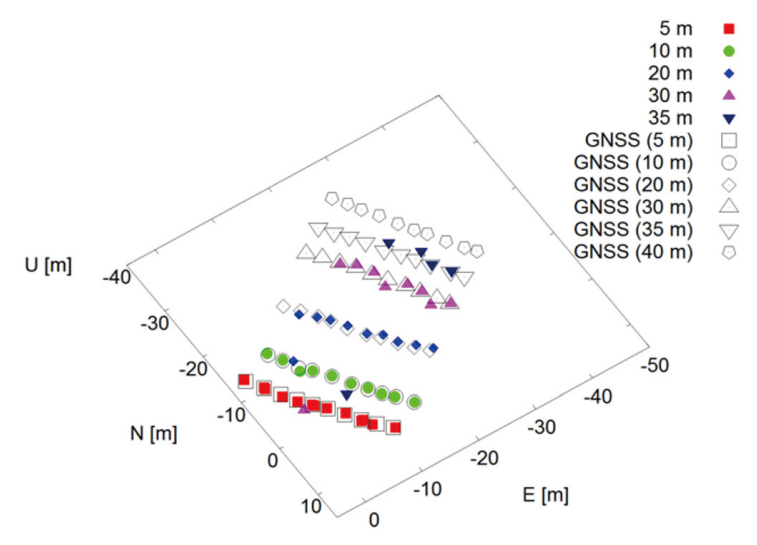

(a) Placement along 6 lines

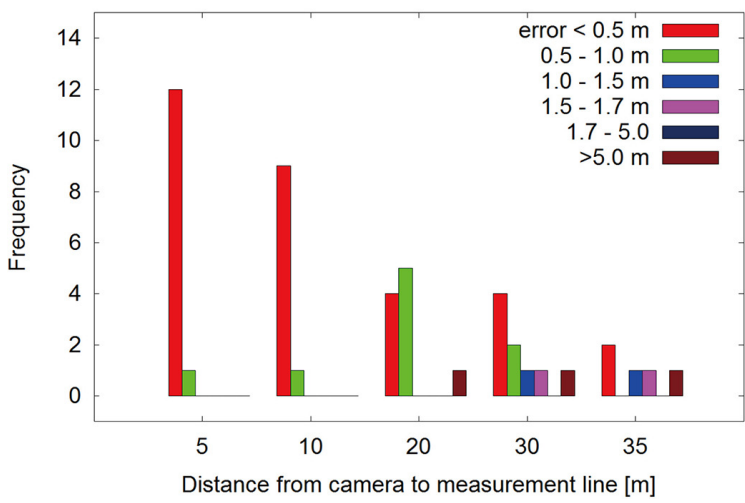

(b) Measurement error histogram

Fig. 6 Results of experiment No. 1: measured positions of 10 markers placed along 6 lines in the ENU coordinate system and measurement error histogram

ment errors were less than $0.5 \mathrm{~m}$ and the maximum error was less than $1.0 \mathrm{~m}$. However, the total number of histogram frequencies at the $5 \mathrm{~m}$ line position was 13 , which was greater than the actual number of 10 , because three markers were classified into not one cluster but two clusters in the nearest neighbor clustering process, and the $3 \mathrm{D}$ position of each marker was measured twice as a redundant measurement. As the line position was set farther from the camera $(20,30$, and $35 \mathrm{~m}$ ) the measurement error became larger ( $>1 \mathrm{~m})$, and one outlier with a measurement error of more than $5 \mathrm{~m}$ was counted in each position of the three lines. However, throughout the experiments, the total number of outliers was only three. The result showed that most of the outliers could be removed robustly by using the voting method that we developed. Within the sensing range of $32.5 \mathrm{~m}$ and with the exception of the three outliers, the RMSE was calculated as $0.47 \mathrm{~m}$, the detection rate was $92.5 \%$, the percentage of measurement errors less than $1 \mathrm{~m}$ was calculated as $95.0 \%(38 /(37+3))$ by including the three redundant measurements mentioned above, and the maximum measurement error was $1.5 \mathrm{~m}$.

The results in experiment No. 1 showed that the marker search system was capable of robustly measuring the 3D position of a marker within a sensing range of approximately $32.5 \mathrm{~m}$ even if a strong wind occasionally blew (some markers were swayed by the wind, and the weather was not good). The results also demonstrated that the marker region extraction method using 3D HSV color histograms and the marker detection method with two types of marker detection filters were able to work robustly and consistently in real time throughout the three days. However, the performance of the system depended on the size of objects having a color similar to the markers.

\section{Results of experiment No. 2}

In experiment No. 2, the $3 \mathrm{D}$ positions of 10 markers (Nos. 1 to 10) placed along the diagonal line were measured 18 times from late morning to late afternoon (Fig. 5 (b)). Figure 7 shows the results of the 3D positions of the 10 markers and the measurement error histogram. Table 5 shows the distance from each marker to the camera, the detection rates, the RMSE, and the maximum error for each marker. As shown in Fig. 7 (a) and Table 5, the 3D positions of markers Nos. 1 to 8 could be measured consistently without any misses in the detection of markers and with no errors larger than $2 \mathrm{~m}$. However, the detection rates of markers Nos. 9 and 10 were both small, $27.8 \%$ and $0 \%$, respectively, because the distances from the two markers to the camera were beyond the upper limit of the marker search system, at $34.2 \mathrm{~m}$ and $39.2 \mathrm{~m}$. The results indicate that the upper limit of the search range is approximately $30 \mathrm{~m}$, the same level as the upper limit in experiment No. 1 .

As shown in Fig. 7 (b), the measurement errors were mostly less than $1 \mathrm{~m}$. In markers Nos. 1 to 8 (markers Nos. 9 and 10 beyond the upper limit of the marker search system excluded), the RMSE was calculated as $0.38 \mathrm{~m}$, the detection rate was $100 \%(144 / 144)$, and the percentage of measurement errors less than $1 \mathrm{~m}$ was $97.9 \%$ (141/144). Furthermore, the maximum measurement error was $1.28 \mathrm{~m}$, no outliners were detected, and the 3D position for each marker was robustly and precisely measured, requiring only one measurement, throughout the experiments. For the nine markers of Nos. 1 to 9 , as the coefficient of correlation between the distance from the marker to the camera and the RMSE was calculated as 0.891 (coefficient of determination 0.793), the positive correlation between the distance and the RMSE indicates that a camera with a high optical zoom magnification needs to be installed to precisely measure the 3D position of a marker in a large field. 


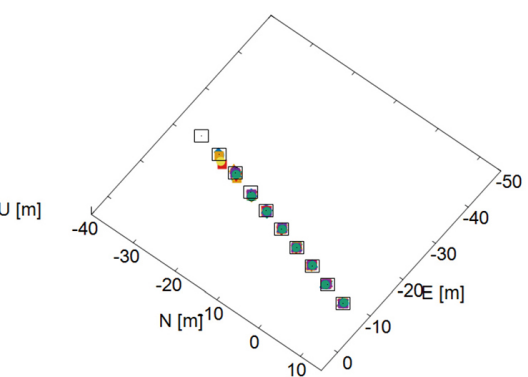

(a) Placement along diagonal line

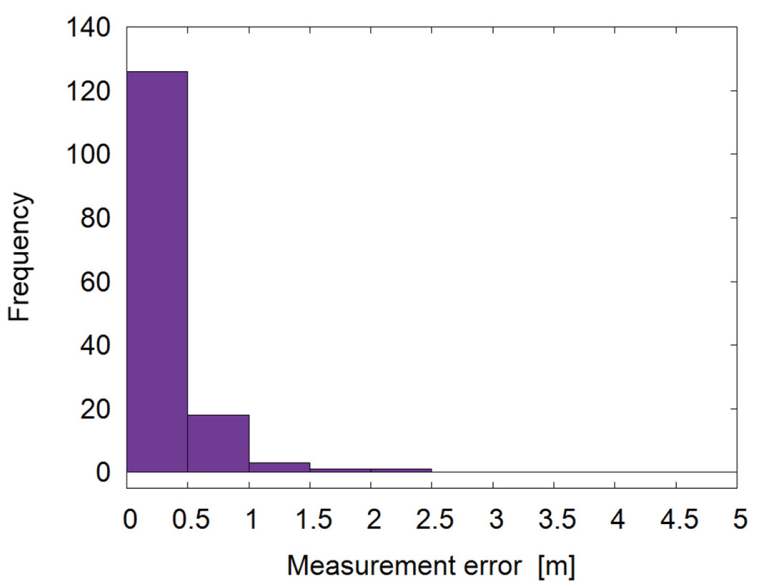

(b) Measurement error histogram

Fig. 7 Results of experiment No. 2: measured positions of 10 markers placed along a diagonal line in the ENU coordinate system and measurement error histogram

The results in experiment No. 2 demonstrate the high robustness and the high accuracy of the marker search system that we developed, with a measurement error of less than $1 \mathrm{~m}$ within a sensing range of approximately $30 \mathrm{~m}$ from late morning to late afternoon. The results also demonstrated the applicability of the marker region extraction method using 3D HSV color histograms and the marker detection method using two types of marker detection filters during the time from late morning to late afternoon. In experiment No. 2, the search time required to scan the entire field was approximately $6 \mathrm{~min}$, 1 min quicker than in experiment No. 1 , because the 10 markers were placed far from the camera as well as near to the camera. However, in both cases, the time required to scan the entire field was long.

\section{Discussion}

Although our developed marker search system was only a prototype, the $3 \mathrm{D}$ positions of markers placed in an agricultural field were measured robustly within a sensing range of approximately $30 \mathrm{~m}$, with an RMSE of less than
Table 5 Detection rates and errors of 10 markers in 18 measurements

\begin{tabular}{ccccc}
\hline No. & $\begin{array}{c}\text { Distance } \\
{[\mathrm{mm}]}\end{array}$ & Detection rate & $\begin{array}{c}\text { RMSE } \\
{[\mathrm{mm}]}\end{array}$ & $\begin{array}{c}\text { Max } \\
{[\mathrm{mm}]}\end{array}$ \\
\hline 1 & 12,811 & $1.00(18 / 18)$ & 141.1 & 295.5 \\
2 & 10,839 & $1.00(18 / 18)$ & 319.6 & 503.1 \\
3 & 10,935 & $1.00(18 / 18)$ & 178.8 & 310.1 \\
4 & 13,024 & $1.00(18 / 18)$ & 174.6 & 355.2 \\
5 & 16,443 & $1.00(18 / 18)$ & 173.9 & 308.1 \\
6 & 20,524 & $1.00(18 / 18)$ & 310.9 & 616.1 \\
7 & 24,839 & $1.00(18 / 18)$ & 699.5 & $1,102.6$ \\
8 & 29,590 & $1.00(18 / 18)$ & 589.6 & $1,276.4$ \\
9 & 34,168 & $0.28(5 / 18)$ & $1,247.8$ & $2,225.5$ \\
10 & 39,186 & $0.00(0 / 18)$ & - & - \\
\hline
\end{tabular}

$0.5 \mathrm{~m}$, without large height differences between the markers and the camera, and without marker deformation. The results demonstrate the possibility that the prototype design can be applied as a worker-robot communication system with multiple color markers and a single PTZ camera. For example, when a worker wants a robot to carry a container full of harvested products out of a field, she / he would be able to send timing and position information of the container to be carried by hanging a marker on a pole or a plant, or by raising a marker in her/his hand so that the marker could be captured by the camera.

However, with the current prototype, several minuets are required to precisely and robustly measure the $3 \mathrm{D}$ positions of multiple markers, even if the markers are not moving. To measure the $3 \mathrm{D}$ positions of moving markers, such as moving workers in uniforms with markers and moving vehicles with markers, the prototype design cannot be applied until the scanning time can be shortened to less than $1 \mathrm{~s}$. In future work, a second prototype will be developed using four PTZ cameras with four focal lengths: $6.7,15.3,23.8$, and $31.1 \mathrm{~mm}$. In the second prototype, as each search grid will be simultaneously observed in real time using multiple focal lengths, the time for measuring the marker position can be shortened while maintaining measurement accuracy. Although the current prototype is not able to measure the marker position before completing the scanning of all of the grids, the second prototype will be designed so that the 3D position of a marker can be measured whenever a marker is detected.

\section{Summary and Conclusions}

In this study, we developed a prototype of an autonomous color marker search system using a single PTZ camera to detect the 3D locations of multiple markers placed in a field. To search for multiple markers over a wide area using a single PTZ camera with $\mathrm{a} \times 10$ optical zoom magnification, 855 
grids were generated in a field. The range of the pan angle of the PTZ camera was set from -90 to $+90^{\circ}$. The pan and tilt angles of the PTZ camera were controlled by considering the horizontal and vertical ranges of the FOV. The search distance was adjusted in eight steps from 4 to $40 \mathrm{~m}$, and the focal length for each gird was controlled by adjusting the zoom value of the PTZ camera so that a marker with a length of $200 \mathrm{~mm}$ in an image was captured at a preset value of 60 pixels.

The marker region in an input image was extracted by using three HSV 3D color histograms: one for the blue of the marker, one for the red of the marker, and one for the white of the uniform. The $2 \mathrm{D}$ position of the marker in the image was detected by using two marker detection filters. The length of the marker, the distance from the marker to the camera, and the pan and tilt angles to capture the marker centered in the image were continuously measured and saved in a buffer memory, while the center of the marker was tracked at a frame rate of 30 frames / $\mathrm{s}$ for $1 \mathrm{~s}$ by using a particle filter and by controlling the pan and tilt angles of the PTZ camera. The $3 \mathrm{D}$ positions of multiple markers were measured in real time in the ENU coordinate system by using a marker distance-anddirection measurement method, a nearest neighbor clustering method, and a novel voting method.

To test the performance of our developed color marker search system, two sets of experiments (Nos. 1 and 2) were conducted using a horizontal cultivated agricultural field $(30 \mathrm{~m}$ wide $\times 50 \mathrm{~m}$ deep). In experiment No. 1 , the 3D positions of 10 markers placed along six measurement lines (approximately $23 \mathrm{~m}$ long) at a point $1.5 \mathrm{~m}$ above the ground were measured in real time in the ENU coordinate system by changing the distance from the camera to the line between 5 , $10,20,30,35$, and $40 \mathrm{~m}$. Despite the fact that some of the markers were occasionally swayed by a strong wind and the sky was not clear at all times, and, in addition, that there were large red and blue objects in the background, within the sensing range of $32.5 \mathrm{~m}$ and with the exception of three outliers, the RMSE was $474.9 \mathrm{~mm}$, the detection rate was $92.5 \%$, the percentage of measurement errors less than $1 \mathrm{~m}$ was $95.0 \%$, and the maximum error was $1,533 \mathrm{~mm}$.

In experiment No. 2 , the 3D positions of 10 markers placed along a diagonal line in a field ( $28 \mathrm{~m}$ wide $\times 35 \mathrm{~m}$ deep) at a point $1.5 \mathrm{~m}$ above the ground were measured in real time 18 times in the ENU coordinate system from late morning to late afternoon. Within the sensing range of $30 \mathrm{~m}$, the RMSE was calculated as $378.9 \mathrm{~mm}$, the detection rate was $100 \%$, the percentage of measurement errors less than $1 \mathrm{~m}$ was $97.9 \%$, the maximum error was $1276.4 \mathrm{~mm}$, and no outliners were detected throughout the day.

The results of the 2 sets of experiments showed that the 3D positions of markers placed in an agricultural field could be measured robustly within a sensing range of approximately $30 \mathrm{~m}$, with an RMSE of less than $500 \mathrm{~mm}$, without a large height difference between markers and the camera, and without marker deformation. The results with the prototype indicated the potential of the design as a tool for communication between a robot and workers.

\section{Acknowledgements}

This study was supported by JSPS (Japan Society for the Promotion of Science) KAKENHI Grant-in-Aid for Scientific Research (C) Number 25450383.

\section{References}

Bagherinia, H. and R. Manduchi. 2013. Robust real-time detection of multi-color markers on a cell phone. Journal of Real-Time Image Processing. 8: 207-223. https://doi.org/10.1007/s11554-011-0206-9, (Accessed 10 June 2021).

Dalal, N. and B. Triggs. 2005. Histograms of oriented gradients for human detection. Proceedings of the 2005 IEEE Conference on Computer Vision and Pattern Recognition (CVPR'05). 1: 886893. San Diego, CA, USA, 20-26 June. https://doi.org/10.1109/ CVPR.2005.177, (Accessed 10 June 2021).

Dalal, N., B. Triggs and C. Schmid. 2006. Human detection using oriented histograms of flow and appearance. Computer Vision ECCV 2006, Lecture Notes in Computer Science, ed. A. Leonardis, H. Bischof and A. Pinz. 3952: 428-441. Germany: Springer-Verlag GmbH. https://doi.org/10.1007/11744047_3, (Accessed 10 June 2021).

Markovic, I., F. Chaumette and I. Petrovic. 2014. Moving object detection, tracking and following using an omnidirectional camera on a mobile robot. Proceedings of the 2014 IEEE International Conference on Robotics and Automation (ICRA). 5630-5635. Hong Kong, China, 31 May-7 June. https://doi.org/ 1109/ICRA.2014.6907687, (Accessed 10 June 2021).

Morio, Y., K. Ibi, K. Kawazu, R. Toyoshima, T. Ohnishi and K. Murakami. 2012. Development of worker position and posture detection system using PTZ camera for intelligent agricultural work assistance. JOURNAL of the JAPANESE SOCIETY of AGRICULTURAL MACHINERY. 74 (4): 301-311. https://doi.org/ 10.11357/jsam.74.301, (Accessed 10 June 2021). (In Japanese).

Morio, Y., T. Shoji and K. Murakami. 2014. Development of agricultural working motion detector to recognize worker behavior. Proceedings of the 7th International Symposium on Machinery and Mechatronics for Agricultural and Biosystems Engineering (ISMAB). 74-80. Yilan, Taiwan, 21-23 May.

Morio, Y., T. Inoue, T. Tanaka and K. Murakami. 2017. Worker posture recognition for understanding agricultural worker behaviors. Journal of Engineering in Agriculture, Environment and Food. 10 (3): 208-222. https://doi.org/10.1016/j.eaef.2017.03.002, (Accessed 10 June 2021).

Shotton, J., A. Fitzgibbon, M. Cook, T. Sharp, M. Finocchio, R. Moore, A. Kipman and A. Blake. 2011. Real-time human pose recognition in parts from single depth images. Proceedings of the IEEE Computer Society Conference on Computer Vision and Pattern Recognition (CVPR2011). 1297-1304. Colorado Springs, CO, USA, 20-25 June. https://doi.org/10.1109/CVPR.2011.5995316, 
(Accessed 10 June 2021).

Tsai, R. Y. 1987. A versatile camera calibration technique for high-accuracy 3D machine vision metrology using off-the-shelf TV cameras and lenses. IEEE Journal on Robotics and Automation. 3 (4): 323-344. https://doi.org/10.1109/JRA.1987.1087109, (Accessed 10 June 2021).
Viola, P. and M. Jones. 2001. Rapid object detection using a boosted cascade of simple features. Proceedings of the IEEE Conference on Computer Vision and Pattern Recognition (CVPR2001). 1: 511-518. Kauai, HI, USA, 8-14 December. https://doi.org/10. 1109/CVPR.2001.990517, (Accessed 10 June 2021).

(Received: 22 May 2015, Accepted: 13 November 2019) 